\title{
Ventricular tachycardia during exercise testing as a predictor of sudden death in patients with chronic chagasic cardiomyopathy and ventricular arrhythmias
}

\author{
Angelo A V de Paola, J Anthony Gomes, Armenio B Terzian, Mauro H Miyamoto, \\ Eulogio E Martinez Fo
}

\begin{abstract}
Objective-To verify the prognostic value of exercise induced ventricular arrhythmias in patients with chagasic cardiomyopathy.

Methods -69 consecutive patients (37 male, 32 female; age range 21-67 years) with chronic chagasic cardiomyopathy and ventricular arrhythmias (more than 10 ventricular premature complexes per hour) were evaluated during treadmill exercise testing, using the Bruce protocol. Protocol end points were peak heart rate or presence of sustained ventricular tachycardia.

Main outcome measure-Sudden cardiac death.

Results-44 patients (group I) developed ventricular tachycardia during exercise testing (five sustained and 39 non-sustained), and 25 did not (group II). After a follow up of 24 (SD 15) months sudden cardiac death occurred in seven patients in group $I$ and in none in group II $(P<$ 0.05).

Conclusions-Ventricular tachycardia on exercise testing is significantly associated with sudden cardiac death in patients with chronic chagasic cardiomyopathy and ventricular arrhythmias.
\end{abstract}

(Br Heart f 1995;74:293-295)

Keywords: chagasic cardiomyopathy, ventricular tachycardia, exercise testing

Division of Clinical Cardiac

Electrophysiology,

São Paulo Federa

University, Escola

Paulista de Medicina,

São Paulo, Brazil

A A V de Paola

A B Terzian

M H Miyamoto

E E Martinez Fo

The Mount Sinai

School of Medicine of

CUNY, The Mount

Sinai Medical Center,

New York, New York,

USA

J A Gomes

Correspondence to:

Dr A A V de Paola, Escola Paulista de Medicina,

Cardiology Division, Rua

Napoleão de Barros 593

São Paulo, Brazil CEP

04024-002.

Accepted for publication

7 December 1994
The prognostic importance of ventricular arrhythmias during exercise testing has been studied in patients with coronary artery disease. ${ }^{7-10}$ The significance of exercise induced arrhythmias has not been reported in Chagas disease. This study was undertaken to verify the prognostic value of exercise induced ventricular arrhythmias in patients with chagasic cardiomyopathy.

\section{Methods}

DEFINITIONS

Chronic chagasic myocarditis was defined as the presence of chronic cardiomyopathy and a positive Machado Guerreiro serum complement and haemaglutinin test.

Ventricular tachycardia was defined as $\geqslant 3$ sequential ventricular complexes at a rate of $>100 / \mathrm{min}$. If this arrhythmia lasted more than 30 seconds or resulted in cardiovascular collapse, it was defined as sustained ventricular tachycardia; if not it was considered to be non-sustained ventricular tachycardia.

PATIENTS, MATERIALS, AND PROCEDURES

The study population included 69 patients with chronic chagasic cardiomyopathy and ventricular arrhythmias. All patients had their antiarrhythmic drugs discontinued for at least five half lives and had more than 10 ventricular premature beats per hour or at least one episode of ventricular tachycardia during a 24 hour Holter monitoring. Three of these patients were on amiodarone therapy (low dose of $200 \mathrm{mg}$ orally per day) and the drug was discontinued for one month before the patient entered the protocol. There were no patients receiving $\beta$ blocker therapy. There were 37 men and 32 women, with ages ranging from 21 to 67 years (mean 46, SD 12, years). Thirty two patients had palpitations, 16 had syncope, and seven had clinical documentation of sustained ventricular tachycardia or ventricular fibrillation. Forty eight patients had a history of congestive heart failure; of these 39 were in New York Heart Association (NYHA) class II, and nine were in class III or IV. Each patient was in a compensated state before entering the protocol.

Echocardiography was performed in all patients and ejection fraction was calculated by the Pombo method. ${ }^{11}$ Patients suspected of having coronary artery disease underwent a coronary angiography study. No patient had any other identifiable organic heart disease.

Treadmill exercise testing-Exercise testing was performed on a motor driven treadmill 
(Model Funbec, ESDO-1), according to the standard Bruce protocol. Twelve-lead ECGs were performed before and after exercise. Leads II and V5 were continuously recorded during exercise and for eight minutes afterwards. The mean recording time for each patient was 17 minutes. Criteria for terminating the exercise were occurrence of maximum heart rate, sustained ventricular tachycardia, or fatigue.

Follow up-Patients were seen in an arrhythmia research clinic by one of the investigators every three months. Mean follow up was 24 (15) months.

\section{STATISTICAL ANALYSIS}

The results are presented as mean (SD). Statistical comparisons were made using the Student $t$ test. Contingency tables were evaluated by $\chi^{2}$ analysis and significance was defined as a probability (P) less than 0.05 using a two tailed analysis.

\section{Results}

The results are shown in the table.

\section{ELECTROCARDIOGRAPHY}

Right bundle branch block, either isolated (six patients) or associated with left anterior hemiblock ( 26 patients) was present in 32 patients $(46 \%)$. Left anterior hemiblock was present in 13 patients (18.9\%), left bundle branch block in four patients $(5 \cdot 8 \%)$, and complete $\mathrm{AV}$ block in the His-Purkinje system in four patients; the latter four patients had VVI pacemakers implanted. Sixteen patients $(23.2 \%)$ did not have conduction disturbances on the surface electrocardiogram.

HOLTER MONITORING

All patients had more than 10 ventricular premature beats per hour; $43 / 69(62 \%)$ had more than 100 per hour; $43 / 69(62 \%)$ had nonsustained ventricular tachycardia; and 32/69 (46\%) had both non-sustained ventricular tachycardia and more than 100 ventricular premature beats per hour.

EJECTION FRACTION

Ejection fraction was estimated by two dimensional echocardiography. Mean ejection frac-

Relation between clinical variables and sudden cardiac death

\begin{tabular}{|c|c|c|c|}
\hline & $n$ & Sudden cardiac death & Significance \\
\hline $\begin{array}{l}\text { CHF class > II } \\
\text { CHF class = I } \\
\text { Syncope } \\
\text { No syncope } \\
\text { Palpitations } \\
\text { No palpitation } \\
\text { Clinical SuVT } \\
\text { No clinical SuVT } \\
\text { Bifascicular block } \\
\text { No bifascicular block } \\
>100 \text { VPC/H on Holter } \\
<100 \text { VPC/H on Holter } \\
\text { EF < 0.40 } \\
\text { EF > 0.40 } \\
\text { VT during ET } \\
\text { No VT during ET }\end{array}$ & $\begin{array}{l}48 \\
21 \\
16 \\
53 \\
32 \\
37 \\
07 \\
62 \\
34 \\
35 \\
43 \\
26 \\
22 \\
47 \\
44 \\
25\end{array}$ & $\begin{array}{l}03(6 \%) \\
04(19 \%) \\
02(12 \%) \\
05(9 \%) \\
04(12 \%) \\
03(8 \%) \\
02(28 \%) \\
05(8 \%) \\
05(14 \%) \\
02(5 \%) \\
04(9 \%) \\
03(11 \%) \\
02(9 \%) \\
05(10 \%) \\
07(16 \%) \\
00\end{array}$ & $\begin{array}{l}\text { NS } \\
\text { NS } \\
\text { NS } \\
\text { NS } \\
\text { NS } \\
\text { NS } \\
\text { NS } \\
\text { P }<0.05\end{array}$ \\
\hline
\end{tabular}

CHF, congestive heart failure; VPC, ventricular premature complexes; EF, left ventricular ejection fraction; SuVT, Sustained ventricular tachycardia; VT, ventricular tachycardia; ET, exercise fraction; tion was $46.6(18.6) \%$. Twenty two patients had an ejection fraction less than $40 \%$ and 47 greater than or equal to $40 \%$.

ARRHYTHMIAS DURING EXERCISE TESTING

Two patients did not have any arrhythmia during exercise; $10(14 \%)$ had isolated premature ventricular beats, $13(18 \%)$ had couplets, $39(56 \%)$ had non-sustained ventricular tachycardia, and five (7\%) had non-sustained and sustained ventricular tachycardia. The four patients with complete AV block were pacemaker dependent during exercise; all of them developed arrhythmias during exercise testing: one couplets, two non-sustained ventricular tachycardia, and one sustained ventricular tachycardia. There was no change in the QTc interval during exercise when compared with control values.

MEDICAL THERAPY AND CLINICAL OUTCOME Patients were seen in an arrhythmia research clinic by one of the investigators every three months. Thirty six patients $(52 \%)$ were off drugs. Antiarrhythmic drugs were prescribed to patients with clinical sustained ventricular arrhythmias, syncope, and inducible ventricular arrhythmias and for those with disabling palpitations. After completing clinical and laboratory evaluation 33 patients $(48 \%)$ were on antiarrhythmic drugs because of symptoms: 23 patients on amiodarone, three on quinidine, three on propafenone, one on amiodarone and propafenone, one on dysopiramide, and two on phenytoin. During a mean follow up of 24 months 12 patients died; seven of these had sudden cardiac death. Of the patients with sudden death, five were on drug therapy (three on amiodarone, one on propafenone, and one on dysopiramide).

Ventricular tachycardia during exercise testing was the only variable that significantly influenced sudden cardiac death in this study population (table).

\section{Discussion}

Regardless of the mechanisms of cardiac arrhythmias the sympathetic nervous system and circulating catecholamines are very important in arrhythmogenesis. The adrenergic state can suppress or provoke cardiac arrhythmias; serious arrhythmias can be provoked or exacerbated by exercise. Also, patients with exercise induced ventricular tachycardia may be more sensitive to plasma noradrenaline than other patients. ${ }^{12}$ The majority of these reports are from patients with coronary heart disease; in some there is a correlation between the occurrence of exercise induced ventricular arrhythmias and significant coronary artery disease and impairment of left ventricular function. ${ }^{1314}$ On the other hand patients with exercise induced non-sustained ventricular tachycardia and normal cardiac function have a good prognosis. ${ }^{15}$ However, the clinical significance of exercise testing in patients with ventricular arrhythmias remains poorly defined.

Chronic chagasic myocarditis is a cardiac 
neuromyopathy with sympathetic ganglion denervation and dysfunction of the parasympathetic autonomic control of the heart. ${ }^{16}$ This gradual autonomic denervation in chronic Chagas cardiomyopathy can partially explain sudden death in some patients. ${ }^{17}$

Our study population was ambulatory and two thirds of them had a left ventricular ejection fraction above $40 \%$ and only $13 \%$ had class III or IV congestive heart failure. These patients with only mild left ventricular dysfunction were different from the usual referral cases, where sustained ventricular tachycardia, disabling symptoms, and severe left ventricular dysfunction were more common. ${ }^{5}$ Additionally, the autonomic dysfunction and the sympathetic reserve of the heart was probably better than in more advanced cases of Chagas cardiomyopathy. Whether the degree of autonomic dysfunction and catecholamine sensitivity explains the results of our study remains unclear. Nonetheless, ventricular tachycardia during exercise testing was the only variable which was significantly associated with sudden cardiac death in our study population with chronic chagasic cardiomyopathy.

The results of our study are applicable to our population with relatively well preserved ventricular function. In this particular subset, exercise testing may be able to select patients who need more aggressive anti-sudden-death therapy. However, the role of inducibility of ventricular tachycardia by programmed stimulation and its suppression with antiarrhythmic drugs needs to be assessed relative to exercise testing in these patients.

1 Podrid PJ, Graboys TB. Exercise stress testing in the management of cardiac rhythm disorders. Med Clin North Am 1984;68:1139-44.

2 Califf RM, McKinnis RA, McNeer F, Harrell FE, Lee KL,
Pryor DB, et al. Prognostic value of ventricular arrhythmias associated with teadmill exercise testing in patients mias associated with treadmill exercise testing in patients ischemic heart disease. $¥ \mathrm{Am}$ Coll Cardiol 1983;2: ischemic
$1060-7$.

3 Prata A. Natural history of chagasic cardiomyopathy. In: Pan American Health Organization, ed. American trypanosomiasis research. Washington, DC: PAHO, 1975: 191.

4 Manço JC, Gallo L, Godoi RA, Fernandes RG, Amorim DS. Degeneration of cardiac nerves in Chagas' disease. Further studies, Circulation 1969;40:879-85.

5 De Paola AAV, Horowitz LN, Myamoto MH, Pinheiro R, Ferreira DF, Terzian AB, et al. Angiographic and electrophysiologic substrates of ventricular tachycardia in patients with chronic chagasic myocarditis. Am $\mathcal{f}$ Cardiol patients with ch

6 Lopes ER, Chapadeiro E. Morte súbita em ărea endêmica da doença de Chagas. Rev Bras Med Trop 1983;16: da-84.

7 Jelinek MV, Lown B. Exercise stress testing for exposure of cardiac arrhythmia. Prog Cardiovasc Dis 1974;16: 497-522.

8 McHenry PL, Morris SN, Kavalier M, Jordan JW. Comparative study of exercise-induced ventricular arrhythmias in normal subjects and patients with documented coronary artery disease. Am $\mathcal{f}$ Cardiol 1976;37: 609-16.

9 Goldschlager N, Cake D, Cohn K. Exercise-induced ventricular arrhythmias in patients with coronary artery disease. Their relation to angiographic findings. $A m \mathcal{F}$ Cardiol 1973;31:434-40.

10 Weiner DA, Levine SR, Klein MD, Ryan TJ. Ventricular arrhythmias during exercise testing: mechanism, arrhythmias during exercise testing: mechanism, response to coronary bypass surgery and
significance. $A m \mp$ Cardiol $1984 ; 53: 1553-7$.

11 Pombo JF, Troy BL, Russell RO. Left ventricular volumes and ejection fraction by echocardiography. Circulation $1971 \cdot 43 \cdot 480-90$

12 Sokoloff NM, Spielman SR, Greenspan AM, Rae AP, Porter RS, Lowenthal DT, et al. Plasma norepinephrine in exercise-induced ventricular tachycardia. $\mathcal{F} \mathrm{Am} \mathrm{Coll}$ Cardiol 1986;8:11-7.

13 Helfant RH, Pine R, Kabde V, Banka VS. Exercise-related ventricular complexes in coronary heart disease. Correlations with ischemia and angiographic severity. Ann Intern Med 1974;80:589-92.

14 McHenry PL, Morris SN, Kavalier M, Jordan JW. Comparative study of exercise-induced ventricular arrhythmias in normal subjects and patients with documented coronary heart disease. Am $₹$ Cardiol 1976;37: mented

15 Fleg JL, Lakatta EG. Prevalence and prognosis of exerciseinduced nonsustained ventricular tachycardia in appar-

16 Manço JC, Gallo L, Godoy RA, Fernandes RG, Amorim Manço JC, Gallo L, Godoy RA, Fernandes RG, Amorim,
DS. Degeneration of the cardiac nerves in Chagas' disease. Circulation 1969;40:879-85.

17 Iosa D, DeQuattro V, Lee DD, Elkayam U, Palmero H. Plasma norepinephrine in Chagas' cardioneuromyopathy: a marker of progressive dysautonomia. Am Heart $\mathcal{f}$ 1989;117:882-7 